\title{
Common Mechanisms in Nephropathy Induced by Toxic Metals
}

\author{
Ivan Sabolić \\ Unit of Molecular Toxicology, Institute for Medical Research and Occupational Health, Zagreb, Croatia
}

\section{Key Words}

Apoptosis $\cdot$ Cadmium $\cdot$ Cisplatin $\cdot$ Heavy metals $\cdot$ Lead $\cdot$

Mercury $\cdot$ Necrosis $\cdot$ Nephrotoxicity

\begin{abstract}
Various metals of unknown function in the body $(\mathrm{Cd}, \mathrm{Cr}, \mathrm{Hg}$, $\mathrm{Pb}, \mathrm{U})$, trace elements in excessive concentrations ( $\mathrm{Co}, \mathrm{Cu}, \mathrm{Fe}$, $\mathrm{Zn}$ ), or metals used in cancer therapy $(\mathrm{Pt}, \mathrm{V})$, accumulate in the mammalian kidney, largely in the proximal tubule (PT) cells, and cause functional and structural damage that results in reabsorptive and secretory defects. The intracellular mechanisms of their toxicity in the PT cells are not well known. Recent studies have indicated an oxidative stress with associated lipid peroxidation, apoptosis, and necrosis as common phenomena in the course of nephrotoxicity of these metals. However, a number of other phenomena, such as the selective inhibition and/or loss of various membrane transporters, enhancement of ion conductances, increased cytoplasmic concentration of calcium, deranged cytoskeleton and cell polarity, impaired endocytosis, swelling and fragmentation of mitochondria, increased expression of metallothionein, heat-shock and multidrug resistance proteins, loss of cell membrane integrity, as well as the damage of mitochondrial and genomic DNAs have been fragmentarily demonstrated for the action of some toxic metals, but their importance for the course of nephrotoxicity and the sequence of events in relation to oxidative stress, apoptosis, and necrosis have not been clearly established. Recent studies of metal toxicity in various tissues and cells of non-renal and renal origin enable us to estimate 'causes and conse-
\end{abstract}

quences' of various phenomena in the metal-induced nephrotoxicity, and to assemble them in a possible common, time-related sequence.

Copyright $\odot 2006$ S. Karger AG, Basel

\section{Introduction}

The mammalian kidney is a structurally and functionally complex organ that plays an important role in control and regulation of homeostasis with various reabsorptive, secretory, metabolic and endocrine functions. Failure to perform these functions is manifested in reabsorptive and secretory defects along the nephron, which in cases of limited malfunctions result in a small molecular weight proteinuria, in more severe cases exhibit also polyuria, glucosuria, aminoaciduria, phosphaturia, and increased excretion of electrolytes, as well as an elevated blood urea nitrogen and creatinine, while in most severe forms, a generalized damage to the kidney functions manifests as the Fanconi syndrome [reviewed in 1]. Whereas some of the functional defects of the nephron are related to the inherited malfunctions of specific genes and/or proteins, a significant number of such cases, particularly in the adult human population, are induced by toxic damages to the nephron following acute (rare event) or chronic (widespread) exposure to various environmental and/or occupational (toxic metals, mycotoxins, pesticides, aristolochic acid, herbicides, etc.) or chemotherapeutic substances (some toxic metals, various drugs used in medicine). 
A variety of metals is nephrotoxic. Some, such as cadmium $(\mathrm{Cd})$, hexavalent chromium $(\mathrm{Cr})$, mercury $(\mathrm{Hg})$, lead $(\mathrm{Pb})$, and uranium $(\mathrm{U})$, have no known functions in the body, whereas some others, like copper $(\mathrm{Cu})$, cobalt $(\mathrm{Co})$, and iron $(\mathrm{Fe})$, represent trace elements that are necessary for the function of various enzymes and other cellular proteins, and become nephrotoxic in case of excessive absorption in the gastrointestinal tract and intracellular accumulation due to inborn errors and deranged metabolism [reviewed in 2]. Here also belongs zinc ( $\mathrm{Zn})$, which in normal concentrations antagonizes the toxicity of other metals, but becomes nephrotoxic in case of overloading. Platinum, usually in form of cisplatin (cisPt), and vanadium $(\mathrm{V})$ have been used in human medicine to treat cancers $[3,4]$, while $\mathrm{V}$ has also been experimentally tested as a promising drug to control diabetes [5].

According to the urinary data, proximal tubules (PT) seem to be the major site of metal-induced nephrotoxicity. Dependent on the severity of intoxication, functional defects are often accompanied with a plethora of structural damages in the PT epithelium, including loss of cell-cell contacts and detachment of cells from the basement membrane, blebbing, shortening and loss of microvilli, loss of basolateral invaginations, vesiculation of the cytoplasm, derangement of the cytoskeleton, swelling, vacuolation and fragmentation of mitochondria, swelling of lysosomes and whole cells, etc. [reviewed in 2, 6, 7].

The mechanisms of metal entry into the PT cells are generally poorly known, and may be different for different metals. For example, the circulated Cd-metallothionein (Cd-MT) and similar metal-protein complexes are filtered in the glomeruli, reabsorbed largely in the proximal convoluted tubules ( $\mathrm{S} 1$ and $\mathrm{S} 2$ segments) by megalin/ cubilin receptor-mediated endocytosis, degraded in the endolysosomal compartment, and the liberated metals are somehow translocated into the cytoplasm, possibly via proton-coupled divalent metal transporter 1 (DMT1) in the endolysosomal membranes $[2,8,9$, and references therein, 10]. Recent studies have indicated that a significant uptake of $\mathrm{Cd}, \mathrm{Hg}, \mathrm{Pb}$, and cisPt in $\mathrm{PT}$ and some other mammalian cells may proceed by ionic and/or molecular homology or 'mimicry', e.g., transported as free ions through calcium $\left(\mathrm{Ca}^{2+}\right)$ channels or ion-transporting proteins $\left(\mathrm{Ca}^{2+}\right.$-ATPase, DMT1, Mn and $\mathrm{Zn}$ transporter ZIP8), or bound to thiol (SH)-containing amino acids and organic anions and cations (methionine, cysteine, homocysteine, N-acetylcysteine (NAC), glutathione $(\mathrm{GSH})$ ), that are carried by specific transporters located in the basolateral (BLM) and/or brush-border membranes (BBM) $[8,11-14]$. The nephrotoxicity following molecular mimicry of metal uptake via organic anion and cation transporters develops predominantly in the straight (S3) portion of $\mathrm{PT}$, reflecting the segment-specific localization of the transporters [13].

After reaching the cytoplasm, most of the toxic metals seem to act by directly hitting various intracellular targets, whereas cisPt has to be metabolically transformed before becoming a nephrotoxin [15]. However, it is assumed that an immediate toxicity, especially during chronic intoxication with small doses of metals, is precluded by the presence of a limited abundance of pre-existing intracellular MT and other $\mathrm{SH}$-rich compounds that bind and sequester some (but not all) toxic metals, and that toxicity starts after this pool of detoxifying substances becomes overwhelmed by the free metal [reviewed in 8,11$]$. Although various nephrotoxic metals can directly damage the integrity of isolated renal PT cell membranes in vitro [16], it is disputable whether they can act similarly in vivo, before entering the cell.

\section{Mechanisms of Metal Nephrotoxicity}

The mechanisms of nephrotoxicity at the cellular level of various toxic metals have been studied for decades, and are still known only in fragments; some more details have been collected for the actions of $\mathrm{Cd}, \mathrm{Hg}$, and cisPt [reviewed in 3, 8, 11]. Recent studies in vivo (experimental animals) and in vitro (renal cortical slices, various cell lines of the renal origin, isolated mitochondria) have indicated oxidative stress, apoptosis, and necrosis as common phenomena in the intracellular action of all toxic metals studied thus far. A number of other phenomena, such as the inhibition of ion channels, ATPases and other transporters, enhancement of ion conductances, increase in intracellular (cytoplasmic) concentration of $\mathrm{Ca}^{2+}\left(\left[\mathrm{Ca}^{2+}\right]_{\mathrm{i}}\right)$ deranged metabolism, cytoskeleton and cell polarity, impaired endocytosis and intracellular vesicle recycling, increased synthesis of MT, upregulation of heat-shock proteins in the cell cytoplasm and multidrug resistance proteins (MDR) in the BBM, selective loss of transporters from the BBM and BLM, destabilization and/or loss of the cell membrane integrity, and distinct structural and functional damage in mitochondria have been demonstrated in fragments in the course of action of $\mathrm{Cd}, \mathrm{Hg}$, and cisPt, but their appearance with other toxic metals have not yet been reported. Also, their importance for the onset and progress of nephrotoxicity, and the sequence of these events in relation to the oxidative stress, apoptosis, and necrosis, have not been clearly 


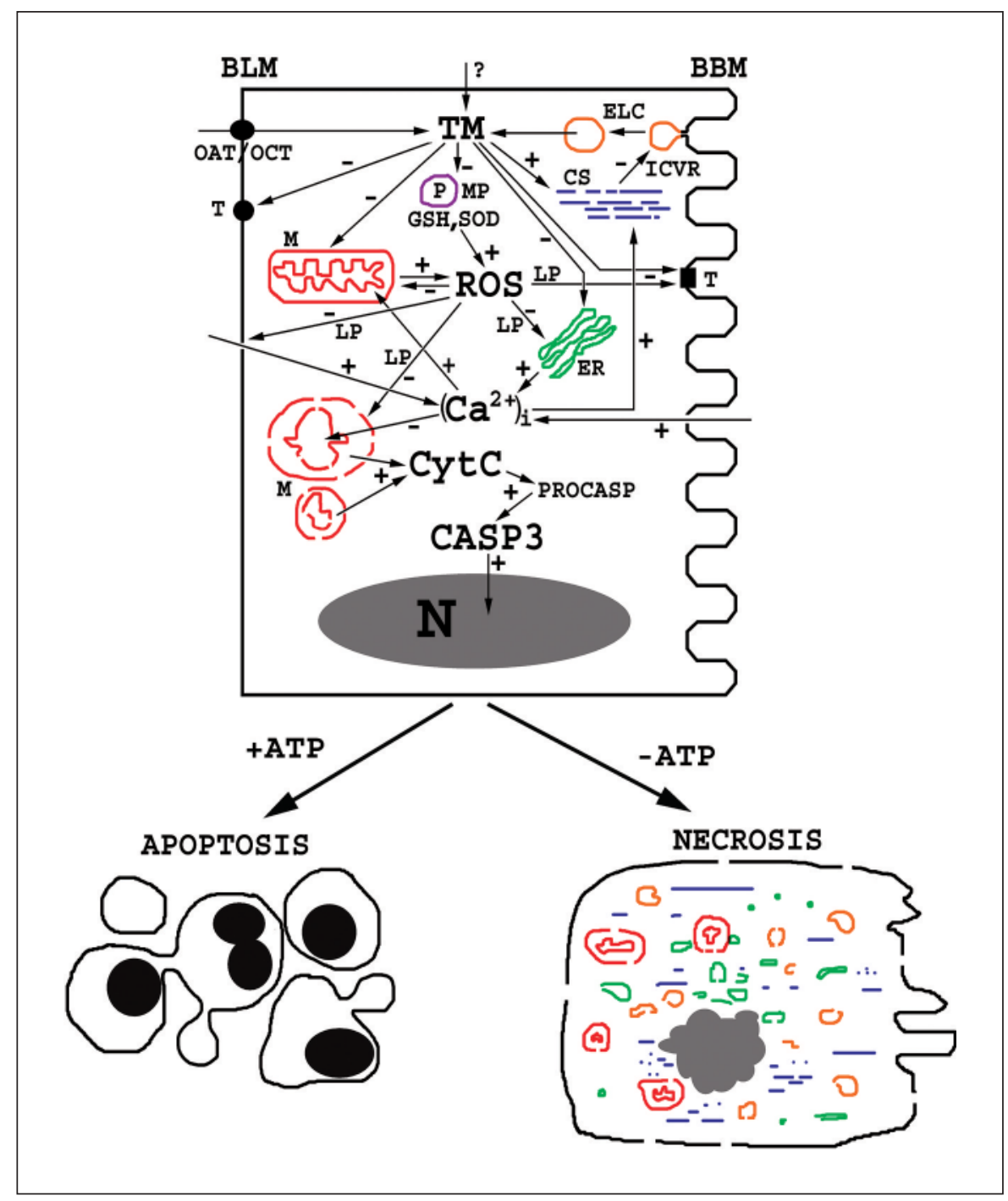

Fig. 1. A simplified scheme of the common mechanisms in metalinduced nephrotoxicity. The toxic metal (TM) can enter the proximal tubule cell via endolysosomal compartment (ELC) at the brush-border membrane (BBM) or via organic anion and cation transporters (OAT/OCT) at the basolateral membrane (BLM), or via some other, less identified mechanisms (?). In the cell, free TM directly inhibit various transport proteins in the plasma membrane $(\mathrm{T})$ and endoplasmic reticulum (ER), polymerization of cytoskeleton (CS), oxidative phosphorylation in mitochondria (M), metabolic processes in peroxisomes (P) and the cytoplasm (MP), and activity of superoxide dismutase (SOD), and deplete reduced glutathione (GSH). The oxidative stress due to diminished elimination and increased production of free radicals leads to the accumulation of reactive oxygen species (ROS). These highly reactive molecules directly or indirectly (via lipid peroxidation (LP)) induce further damage to the function/permeability of various intracellular organelles, leading to the cytoplasmic accumulation of $\mathrm{Ca}^{2+}{ }_{\mathrm{i}}$. The elevated $\mathrm{Ca}^{2+}{ }_{\mathrm{i}}$ promotes a variety of intracellular reactions, including depolymerization of the cytoskeleton, thus contributing to the diminished endocytosis and intracellular vesicle recycling (ICVR). Synergistic action of ROS and $\mathrm{Ca}^{2+}{ }_{\mathrm{i}}$ leads to the damage of mitochondrial (M) function, organelle swelling and release of cytochrome $\mathrm{c}(\mathrm{CytC})$ and other proteins via ruptured membranes into the cytoplasm, where it promotes transformation of inactive procaspases (PROCASP) into caspases. The final, executive caspase 3 (CASP3) translocates the 'cell death message' into the nucleus $(\mathrm{N})$. The cell death may proceed by apoptosis, if the mitochondrial ATP synthesis is still active (+ATP), or by necrosis, if ATP is heavily depleted (-ATP). For more details, see the text. Effects labeled with '-' indicate inhibition, whereas effects labeled with ' + ' indicate stimulation of the activity/process. 
established. However, by comparing the data obtained in recent studies of metal toxicity in various tissues and cells of non-renal and renal origin enable us to estimate 'causes and consequences' of various phenomena that occur in the metal-induced nephrotoxicity, and to assemble them in a possible common, time-related pattern (fig. 1).

An initial, common event in the action of all toxic metals in the PT cells studied thus far seems to be generation of oxidative stress, that is manifested by: (a) depletion of intracellular antioxidants (largely GSH) and free radical scavengers (vitamins $\mathrm{E}$ and C), (b) inhibition of the activity of various enzymes that contribute to the metabolism and detoxication of free radicals (reactive oxygen species (ROS)), such as GSH-peroxidase, GSH-reductase, GSHtransferase, catalase and superoxide dismutase (SOD), and (c) increased production of ROS (superoxide anion radical, hydrogen peroxide, peroxyl radical, hydroxyl radical, nitric oxide, peroxynitrite radical, etc.). In intact cells, a limited amount of ROS is produced in normal metabolic processes in the cytoplasm and peroxisomes, whereas a bulk of these substances is generated as a side product during oxidative phosphorylation in mitochondria [reviewed in 17-19]. In subtoxic concentrations these products may act as second messengers in some of the intracellular signal transduction pathways [reviewed in 20] or are buffered by GSH, the main scavenger of ROS in the cell cytoplasm and mitochondria, and by other intracellular antioxidants, or further metabolized by metalloenzyme SOD into less damaging molecules. However, having a high affinity for $\mathrm{SH}$ groups, free toxic metals can directly bind to cellular antioxidants and related enzymes, and inhibit buffering and detoxication of ROS. Some redox-active metals, such as $\mathrm{Fe}, \mathrm{Cu}$, and $\mathrm{Cr}$, directly catalyze generation of hydroxyl radical from hydrogen peroxide (Fenton reaction) and other ROS $[17,18$, and references therein], whereas the redox-inactive metals, such as $\mathrm{Cd}, \mathrm{Hg}$, and cisPt, diminish the ROS-scavenging capacity in the cells, but may also stimulate the generation of ROS indirectly - by displacing Feand $\mathrm{Cu}$ from MT and other metal-containing cellular proteins, which then may accelerate production of ROS via Fenton reaction [21-23].

Numerous studies in non-renal experimental models of metal toxicity [18, and references therein, 24], and in various models of nephrotoxicity induced with $\mathrm{Cd}, \mathrm{Hg}$, cisPt, Cr, Co, and V [reviewed in 3, 8, 11, 25, 26], showed that the elevated concentrations of ROS affect a number of cellular ion transport pathways via: (a) modulation of the activity of various ion transport proteins by changing (oxidizing) the redox state of intracellular space and/or directly oxidizing their functionally-important $\mathrm{SH}$ groups, (b) peroxidation of membrane phospholipids that could change the microenvironment necessary for optimal function of the transporters and increase membrane permeability for $\mathrm{Ca}^{2+}$ and other ions, (c) altering transmembrane signaling by inhibiting regulatory enzymes, and (d) decreasing cellular ATP levels by inhibiting oxidative phosphorylation in mitochondria. While some of these effects may be caused by direct binding of toxic metals to the respective proteins and phospholipids, in many models of metal (nephro)toxicity the induction of oxidative stress was thus far demonstrated only indirectly, by finding an increased abundance of peroxidized lipids, as estimated from an enhanced production of malondialdehyde and thiobarbituric acid-reactive substances in tissues and cells $[3,8,11$, and references therein]. However, recent studies have shown that mitochondria from the mammalian kidney and other organs are a direct target for $\mathrm{Cd}$ and some other toxic metals. Metals rapidly accumulate in this organelle, possibly via $\mathrm{Ca}^{2+}$ uniporter in the inner mitochondrial membrane, bind to the electron transfer proteins and inhibit oxidative phosphorylation, causing hyperproduction of ROS $[24,27-29]$. Studies have also shown that in toxic conditions due to exposure to some toxic metals and other xenobiotics mitochondria are not only the major producer of, but are also the major target for ROS [reviewed in 19, 30-33]. The locally generated oxidative radicals further damage the mitochondrial function and structure by: (a) depleting the pool of matrix GSH and thus shifting the redox status of the matrix into more oxidized direction, (b) oxidizing respiratory enzymes and inhibiting their activity, (c) peroxidizing cardiolipin, a major lipid and cytochrome c-anchoring component of the inner mitochondrial membrane, (d) dissipating transmembrane potential and ionic gradients via triggering $\mathrm{Ca}^{2+}$-assisted formation of mitochondrial permeability transition pore (see later), and (e) causing mitochondrial DNA mutations, fragmentations, and deletions. In most of these studies the symptoms of oxidative stress, ROS production, peroxidation of lipids, and their deleterious consequences to the cell and mitochondrial structure and function were ameliorated or prevented by treating animals/tissue slices/cell lines/isolated mitochondria with one or more free radical scavengers and/or antioxidants (GSH, NAC, melatonin, vitamins A, E and C, selenium, mannitol, aspirin, glycine, glutamine, L-carnitine, dimethylthiourea, pyruvate, lipoic or caffeic acid, Trolox, various bioflavonoids (catechin, quercetin, curcumin, 
erdosteine, phytoestrogens, tannin, G-rutin)), thus indicating ROS as the crucial, initial trigger of all further damaging processes.

Although a number of ion channels, pumps, cotransporters and exchangers in the kidney tissue/cells/isolated membranes can be directly inhibited by various toxic metal or ROS, or diminished by lysosomal and proteasomal degradation [reviewed in $9,11,18,26,34$ ], or perturbed in activity due to oxidative stress-mediated shift of the cellular redox status to a more oxidized state, an effect of these compounds upon mechanisms that control intracellular $\mathrm{Ca}^{2+}$ homeostasis seems to be of paramount importance to the further changes in cell function and structure. Studies have shown that the dysfunction of intracellular $\mathrm{Ca}^{2+}$ homeostasis is manifested as a dramatic increase in $\left[\mathrm{Ca}^{2+}\right]_{\mathrm{i}}$ caused by: (a) opening of $\mathrm{Ca}^{2+}$ channels in endoplasmic reticulum and plasma membranes via activation of inositol-1,4,5-triphosphate ( $\left.\mathrm{InsP}_{3}\right)$-mediated cell signaling, (b) direct inhibition and/or loss of $\mathrm{Ca}^{2+}$ transporters $\left(\mathrm{Ca}^{2+}\right.$-ATPases, $\mathrm{Na}^{+} / \mathrm{Ca}^{2+}$ exchanger) in the plasma membrane and sarcoplasmic reticulum, (c) displacement of $\mathrm{Ca}^{2+}$ from the intracellular binding proteins, and (d) release of $\mathrm{Ca}^{2+}$ from the intracellular stores (various organelles including mitochondria) and its influx from the extracellular space via ROS-, peroxidized lipid-, and/or metal-induced increased permeability of intracellular organelles and the plasma membrane [20, 32, 35-37, and references therein]. A rapid mobilization of $\left[\mathrm{Ca}^{2+}\right]_{\mathrm{i}}$ via the $\mathrm{InsP}_{3}$-mediated signaling mechanism was shown for $\mathrm{Cd}$ and other divalent metals in cultured fibroblasts [38], while a significant mobilization of $\left[\mathrm{Ca}^{2+}\right]_{\mathrm{i}}$ in the renal tissue and cells has thus far been demonstrated for $\mathrm{Cd}, \mathrm{Hg}$, and cisPt [11,26, and references therein, 39].

Studies in different experimental models have shown that elevation of $\left[\mathrm{Ca}^{2+}\right]_{\mathrm{i}}$ has most serious consequences for the integrity of cytoskeleton, intracellular vesicle recycling, cell polarity, and for further processes that affect mitochondrial structure and function, and in ultima linea initiate cell death via apoptosis or necrosis [30-32, 35, and references therein]. Sustained increase in $\left[\mathrm{Ca}^{2+}\right]_{\mathrm{i}}$ disrupts the cytoskeletal network and activates various $\mathrm{Ca}^{2+}$-dependent catabolic enzymes, such as phospholipase $\mathrm{A}_{2}$ and other phospholipases, proteases (calpains), and endonucleases. $\mathrm{Ca}^{2+}$ itself, or via $\mathrm{Ca}^{2+}$-activated proteases, strongly promotes disassembly of microtubules and dissociation of actin microfilaments from their anchoring proteins in the plasma membrane. In addition, studies in cell-free systems and in various non-renal and renal cell lines have shown that $\mathrm{Cd}, \mathrm{Hg}, \mathrm{Pb}, \mathrm{Cr}, \mathrm{Cu}$, and
cisPt can directly interfere with polymerization of microtubules and actin filaments, probably by binding to the $\mathrm{SH}$ groups in the monomeric proteins. The breakdown of the cytoskeleton network causes blebbing of the plasma membrane [40], and, as shown in our recent studies with CdMT in rats in vivo [6], in PT cells it causes impaired intracellular vesicle recycling, selective removal of some brush-border transporters and their translocation into the basolateral membrane, and loss of cell polarity. Similar $\mathrm{Ca}^{2+}$-dependent processes may also account for the increased molecular mobility in the lipid phase and strongly increased $\mathrm{K}^{+}$conductance in the renal cortical BBM from Cd-treated rats [41].

Recent studies have shown that in intact cells the mitochondrial respiration and maintenance of the membrane potential are tightly regulated by the $\mathrm{Ins}_{3}$ signaling-mediated pulsatile nature of $\left[\mathrm{Ca}^{2+}\right]_{i}[$ reviewed in 42 , 43]. Under physiological conditions, $\mathrm{Ca}^{2+}$ is accumulated in the mitochondrial matrix via a coordinated action of voltage-dependent $\mathrm{Ca}^{2+}$ channel in the outer membrane and the membrane potential-driven $\mathrm{Ca}^{2+}$ uniporter in the inner membrane, whereas $\mathrm{Ca}^{2+}$ efflux is largely mediated by the $\mathrm{Na}^{+} / \mathrm{Ca}^{2+}$ antiport in the inner membrane. Inside the matrix, $\mathrm{Ca}^{2+}$ is a positive effector of ATP synthesis via the Krebs cycle and oxidative phosphorylation; a limited increase in the matrix $\mathrm{Ca}^{2+}$ concentration accelerates respiration and ATP production. However, in various toxic conditions the massive and sustained overloading with $\mathrm{Ca}^{2+}$ becomes detrimental to the mitochondrial function and structure because it promotes additional ROS formation by structural alterations of the inner mitochondrial membrane via disorganizing the assembly of lipids (largely cardiolipin) and respiratory chain proteins, and stimulates the ROS-mediated oxidation of the SH-rich inner and outer mitochondrial membrane proteins, causing their cross-linkage and aggregation, thus accelerating formation of the non-specific, cyclophylin-mediated and cyclosporin A-inhibitable mitochondrial permeability transition pore. This pore is highly permeable to protons, water, and other ions and small molecules of $<1,500 \mathrm{Da}$. A possible role of water channel AQP8, that is present in the inner mitochondrial membrane [44] and found to be upregulated by Cd treatment in mitochondria from the rat renal cortex [29], in these processes has not been clarified. The loss of ionic/ osmotic gradients leads to dissipation of mitochondrial transmembrane potential, heavily disrupted oxidative phosphorylation and ATP synthesis, organelle swelling and rupture of its membranes, fragmentation, detachment of cytochrome c from cardiolipin and a release of 
this and other mitochondrial proteins into the cytoplasm $[29,32,34,42,43$, and references therein].

In a variety of experimental models of metal-induced nephrotoxicity, oxidative stress and its immediate intracellular consequences described above can lead to the PT cell death by apoptosis or necrosis. Dependent on the concentration of applied toxic metal and/or extent and duration of the injury (elevation of ROS and cytoplasmic/ mitochondrial $\mathrm{Ca}^{2+}$ concentration), the mitochondrial ATP-producing machinery may determine the apoptotic or necrotic fate of the cell; small concentrations of Cd or some other toxic metal, which produce less ROS and incompletely inhibit oxidative phosphorylation, favor apoptosis, whereas sudden exposition to high concentrations of toxic metals, or their significant intracellular accumulation after a long-term exposition to lower concentrations, may induce an oxidative injury with massive breakdown of cellular metabolism, strong increase in cytoplasmic and mitochondrial $\mathrm{Ca}^{2+}$, dramatic inhibition of mitochondrial function and ATP depletion, that result in necrosis [reviewed in 9, 30-32].

Apoptosis, or programmed cells death, is initiated by the $\left(\mathrm{ROS}+\mathrm{Ca}^{2+}\right)$-mediated release of cytochrome $\mathrm{c}$ and several other mitochondrial proteins into the cytoplasm, where in complex reactions they activate a cascade of specific proteases (caspases), including the caspase 3, that executes the final apoptotic processes in the nucleus that result in DNA and cell fragmentation [reviewed in 3032]. Apoptosis is an energy-dependent and gene-regulated process, morphologically characterized by cell membrane blebbing, nuclear condensation and fragmentation, chromatin aggregation, coarse degradation of the genomic DNA, cell shrinkage and fragmentation into spherical apoptotic bodies, that are finally digested by adjacent cells and macrophages, without inflammation. Although apoptosis can be triggered by the processes in $\mathrm{mi}$ tochondria (intrinsic pathway) and/or receptor-mediated signaling through death receptors on the cell membrane (extrinsic pathway), and both of them were demonstrated in some models of metal-induced nephrotoxicity $(\mathrm{Cd}$, cisPt), the oxidative stress-related mechanisms that trigger mitochondrial processes and are inhibited or prevented by free radical scavengers and antioxidants may be the predominant mode of inducing apoptosis in various models of metal nephrotoxicity $[9,25,30-32,35$, and references therein]. Necrosis seems to use the biochemical pathways identical to those in apoptosis which, however, have lost regulations due to heavy depletion of the energy. In its developed stage, necrosis is morphologically characterized by the loss of cell membrane integrity, cytoplas- mic swelling, vacuolation of mitochondria, nuclear pycnosis, release of cell content and lysosomal enzymes in the surrounding extracellular space, causing local inflammation [reviewed in 30,31]. In experimental animals treated with usual nephrotoxic doses of $\mathrm{Cd}, \mathrm{Hg}$, or cisPt, both apoptotic and necrotic events can be detected in the same kidney tissue.

\section{Mechanisms of Protection from Cell Death in Metal Nephrotoxicity}

If not immediately killed by apoptosis or necrosis, in case of metal intoxication the PT cells express a battery of short- and long-term effects that can be activated, favoring the cell survival. Short-term effects include: (a) release of $\mathrm{Zn}$, displaced by the toxic metal from the preexisting intracellular pool of Zn-MT; the liberated Zn can transiently inhibit the activity of metabolic enzymes, protect free $\mathrm{SH}$ groups from oxidation, stabilize membranes, protect various $\mathrm{Zn}$-dependent proteins in the cytoplasm and $\mathrm{Zn}$-finger proteins in the nucleus, and potently inhibit caspase 3 and $\mathrm{Ca}^{2+}$-dependent endonucleases, both critical for finishing apoptosis [reviewed in 45]; (b) inhibition/loss of $\mathrm{H}^{+}$-ATPase and $\mathrm{Na}^{+} / \mathrm{H}^{+}$-exchanger $\mathrm{NHE}_{3}$ from the BBM in PT cells, as detected in $\mathrm{Cd}$ - and cisPt-treated rats [reviewed in 32,46], may result in impaired extrusion of protons and intracellular acidosis, which diminishes apoptosis by inhibiting the activity of degradative enzymes and permeability of the mitochondrial membrane [47]; (c) mobilization of antiapoptotic protein Bcl-2 from intracellular membranes, which inhibits release of cytochrome $\mathrm{c}$ from mitochondria, and thus interrupts the apoptotic process [30,31, 47, and references therein]. Long-term effects include: (a) increased synthesis of MT via metal-response and antioxidant-response elements present in the MT gene promoter [48]; MT acts as a multifunctional stress protein induced by various toxic metals that restricts their toxicity by binding them, and also serves as an efficient scavenger of hydroxyl radicals, (b) increased abundance of GSH and SOD after prolonged oxidative stress induced with some toxic metals in rat kidney [reviewed in 49], (c) induction of heme oxidase in the kidney, associated with increased renal content of heme and ferritin in cisPT nephrotoxicity [50], which leads to the binding and detoxication of the redox-active Fe and thus decreases the Fenton reaction-mediated production of ROS, (d) upregulation of MDR proteins in the BBM of the PT cell line by Cd [9, and references therein] and of the kidney PT in rats treat- 
ed with $\mathrm{Cd}, \mathrm{Hg}, \mathrm{Pb}$, and cisPt [pers. unpubl. data]; these ATP-driven drug efflux pumps may extrude peroxidized lipids and various other toxic compounds generated during oxidative stress, and (e) induction of various protective proteins ( $\mathrm{p} 38$, heat-shock proteins) that interfere with the process of apoptosis and/or contribute to amelioration of various, oxidative stress-induced intracellular injuries [31, 32]. In most cases, these protective mechanisms were also more or less attenuated or prevented by free radical scavengers and antioxidants, indicating oxidative stress and ROS as major causes of their induction.

\section{Conclusion}

Oxidative stress, apoptosis and necrosis appear as common mechanisms in various in vivo and in vitro models of nephrotoxicity induced by toxic metals. These phenomena, that take place largely in PT, can be more or less corrected or prevented by free radical scavengers and antioxidants, indicating oxidative stress and ROS as 'the mother of most (if not all) problems'. Most of these phenomena resemble those in the hypoxia-induced PT damages, which can also be corrected with antioxidants and free radical scavengers [51]. These facts point to the possible strategy to common therapy of such renal injuries where the intracellular uptake of toxic metals or other toxic substances would be adequately prevented by the relevant inhibitors (chelators), whereas the ROS generation and ROS-mediated processes would be prevented or ameliorated by relevant antioxidants and scavengers of free radicals and $\mathrm{Fe}$.

\section{Acknowledgements}

This review is based on the data collected from more than 200 research papers and review articles that have been published in this field since 1985; I apologize for not being able to cite all of them due to space limitation. I thank Prof. Dr. Krista KostialŠimonović for critical reading of the manuscript. This work was supported by grant No. 0022011 (Ministry for Science, Education and Sport, Republic of Croatia).

\section{References}

1 Bergeron M, Goodyear PR, Gougoux A, Lapointe JY: Pathophysiology of renal hyperaminoacidurias and glucosuria; in Seldin DW, Giebisch G (eds): The Kidney, Physiology and Pathophysiology, ed 3. Philadelphia, Lippincott Williams \& Wilkins, 2000, vol 2, pp 2211-2233.

2 Dock L, Vahter M: Metal toxicology; in Ballantyne B, Marrs T, Syversen T (eds): General and Applied Toxicology, ed 2. London, Macmillan Reference Ltd, 1999, vol 3, pp 2049-2078.

-3 Arany I, Safirstein RL: Cisplatin nephrotoxicity. Semin Nephrol 2003;23:460-464.

4 Evangelou AM: Vanadium in cancer treatment. Crit Rev Oncol Hematol 2002;42:249265.

5 Domingo JL: Vanadium and tungsten derivatives as antidiabetic agents - a review of their toxic effects. Biol Trace Element Res 2002;88:97-112.

-6 Sabolic I, Ljubojevic M, Herak-Kramberger CM, Brown D: Cd-MT causes endocytosis of brush-border transporters in rat renal proximal tubules. Am J Physiol Renal Physiol 2002;283:F1389-F1402.

7 Sabolic I, Herak-Kramberger CM, Antolovic $\mathrm{R}$, Breton S, Brown D: Loss of basolateral invaginations in proximal tubules of cadmium-intoxicated rats is independent of microtubules and clathrin. Toxicology 2006;218: 149-163.

\footnotetext{
$>8$
} Zalups RK, Ahmad S: Molecular handling of cadmium in transporting epithelia. Toxicol Appl Pharmacol 2003;186:163-188.

$\checkmark 9$ Thevenod F: Nephrotoxicity and the proximal tubule. Nephron Physiol 2003;93:8793.

10 Abouhamed M, Gburek J, Liu W, Torchalski B, Wilhelm A, Wolff NA, Christensen EI, Thevenod F, Smith CP: Divalent metal transporter 1 in the kidney proximal tubule is expressed in late endosomes/lysosomal membranes: implications for renal handling of protein-metal complexes. Am J Physiol Renal Physiol 2006;290:F1525-F1533.

11 Zalups RK: Molecular interactions with mercury in the kidney. Pharmacol Rev 2000; 52:113-143.

12 Ludvig T, Reithmuller C, Gekle M, Schwerdt G, Oberleithner H: Nephrotoxicity of platinum complexes is related to basolateral organic cation transport. Kidney Int 2004;66: 196-202.

13 Bridges CC, Zalups RK: Molecular and ionic mimicry and the transport of toxic metals. Toxicol Appl Pharmacol 2005;204:274-308.

-14 Dalton TP, He L, Wang B, Miller ML, Jin L, Stringer KF, Chang X, Baxter CS, Nebert DW: Identification of mouse SLC39A8 as the transporter responsible for cadmium-induced toxicity in the testis. Proc Natl Acad Sci USA 2005;102:3401-3406.
15 Townsend DM, Deng M, Zhang L, Lapus MG, Hanigan MH: Metabolism of cisplatin to a nephrotoxin in proximal tubule cells. J Am Soc Nephrol 2003;14:1-10.

16 Herak-Kramberger CM, Sabolic I: The integrity of renal cortical brush-border and basolateral membrane vesicles is damaged in vitro by nephrotoxic heavy metals. Toxicology 2001;156:139-147.

17 Bergendi L, Benes L, Durackova Z, Ferencik M: Chemistry, physiology and pathology of free radicals. Life Sci 1999;65:1865-1874.

18 Kourie JI: Interaction of reactive oxygen species with ion transport mechanisms. Am J Physiol Cell Physiol 1998;275:C1-C24.

19 Turrens JF: Mitochondrial formation of reactive oxygen species. J Physiol 2003;552: 335-344.

20 Suzuki YJ, Forman HJ, Sevanian A: Oxidants as stimulators of signal transduction. Free Radic Biol Med 1997;22:269-285.

$\checkmark 21$ Koizumi T, Li ZG: Role of oxidative stress in single dose, cadmium-induced testicular cancer. J Toxicol Environ Health 1992;37: 25-36.

22 Casalino E, Sblano C, Landriscina C: Enzyme activity alteration by cadmium administration to rats: the possibility of iron involvement in lipid peroxidation. Arch Biochem Biophys 1997;346:171-179. 
-23 Yiin SJ, Chern CL, Sheu JY, Tseng WC, Lin $\mathrm{TH}$ : Cadmium-induced renal lipid peroxidation in rats and protection by selenium. J Toxicol Environ Health A 1999;57:403-413.

-24 Pourahmad J, O’Brien PJ, Jokar F, Daraei B: Carcinogenic metal induced sites of reactive oxygen species formation in hepatocytes. Toxicol In Vitro 2003;17:803-810.

25 Boobis AR, Fawthrop DJ, Davies DS: Mechanisms of cell death. Trends Pharmacol Sci 1989;10:275-280.

-26 Zhang JG, Lindup WE: Cisplatin-induced nephrotoxicity in vitro - increases in cytosolic calcium concentration and the inhibition of cytosolic and mitochondrial protein kinase C. Toxicol Lett 1996;89:11-17.

-27 Tang W, Shaikh ZA: Renal cortical mitochondrial dysfunction upon cadmium metallothionein administration to SpragueDawley rats. J Toxicol Environ Health A 2001;63:221-235.

-28 Wang Y, Fang J, Leonard SS, Rao KMK: Cadmium inhibits the electron transfer chain and induces reactive oxygen species. Free Radic Biol Med 2004;36:1434-1443.

-29 Lee WK, Bork, U, Gholamrezaei F, Thevenod $\mathrm{F}$ : $\mathrm{Cd}^{2+}$-induced cytochrome $\mathrm{c}$ release in apoptotic proximal tubule cells: role of mitochondrial permeability transition pore and $\mathrm{Ca}^{2+}$ uniporter. Am J Physiol Renal Physiol 2005;288:F27-F39.

>30 Susin SA, Zamzami N, Kroemer G: Mitochondria as regulators of apoptosis: Doubt no more. Biochim Biophys Acta 1998;1366: 151-165.

-31 Padanilam BJ: Cell death induced by acute renal injury: a perspective on the contributions of apoptosis and necrosis. Am J Physiol Renal Physiol 2003;284:F608-F627.
32 Brookes PS, Yoon Y, Robotham JL, Anders MW, Sheu SS: Calcium, ATP, and ROS: a mitochondrial love-hate triangle. Am J Physiol Cell Physiol 2004;287:C817-C833.

33 Lenaz G: Role of mitochondria in oxidative stress and ageing. Biochim Biophys Acta 1998;1366:53-67.

34 Kowaltowski AJ, Vercesi AE: Mitochondrial damage induced by conditions of oxidative stress. Free Radic Biol Med 1999;26:463471.

35 Orrenius S, McConkey DJ, Bellomo G, Nicotera P: Role of $\mathrm{Ca}^{2+}$ in toxic cell killing. Trends Pharmacol Sci 1989;10:281-285.

36 Ermak G, Davies KJA: Calcium and oxidative stress: from cell signalling to cell death. Mol Immunol 2001;38:713-721.

37 Hajnoczki G, Davies E, Madesh M: Calcium signalling and apoptosis. Biochem Biophys Res Commun 2003;304:445-454.

38 Smith JB, Dwyer SD, Smith L: Cadmium evokes inositol polyphosphate formation and calcium mobilization. J Biol Chem 1989; 264:7115-7118.

$>39$ Yeh JH, Chung HM, Ho CM, Jan CR: Mercury-induced $\mathrm{Ca}^{2+}$ increase and cytotoxicity in renal tubular cells. Life Sci 2004;74:20752083.

40 Jewell SA, Bellomo G, Thor H, Orrenius S, Smith M: Bleb formation in hepatocytes during drug metabolism is caused by disturbances in thiol and calcium ion homeostasis. Science 1982;217:1257-1259.

41 Zuvic-Butorac M, Herak-Kramberger CM, Krilov D, Sabolic I, Herak JN: EPR study of lipid phase in renal cortical membrane organelles from intact and cadmium-intoxicated rats. Biochim Biophys Acta 2005;1718: 44-52.

-42 Szalai G, Krishnamurthy R, Hajnoczky G: Apoptosis-driven by $\mathrm{IP}_{3}$-linked mitochondrial calcium signals. EMBO J 1999;18 6349-6361.
43 Duchen MR: Mitochondria and calcium: from cell signalling to cell death. J Physiol 2000;529:57-68.

44 Calamita G, Ferri D, Gena P, Liquori GE, Cavalier A, Thomas D, Svelto M: The inner mitochondrial membrane has aquaporin-8 water channels and is highly permeable to water. J Biol Chem 2005;280:17149-17153.

45 Perry DK, Smyth MJ, Stennicke HR, Salvesen GS, Duriez P, Poirier GG, Hannun YA: Zinc is a potent inhibitor of the apoptotic protease, caspase 3. J Biol Chem 1997;272: 18530-18533.

46 Shiraishi Y, Nagai J, Murakami T, Takano M: Effect of cisplatin on $\mathrm{H}^{+}$transport by $\mathrm{H}^{+}$ATPase and $\mathrm{Na}^{+} / \mathrm{H}^{+}$exchanger in rat renal brush-border membrane. Life Sci 2000;67: 1047-1058.

47 Kim JS, He L, Lemaster JL: Mitochondrial permeability transition: common pathway to necrosis and apoptosis. Biochem Biophys Res Commun 2003;304:463-470.

48 Andrews GK: Regulation of metallothionein gene expression by oxidative stress and metal ions. Biochem Pharmacol 2000;59:95104.

49 Shaikh ZA, Vu TT, Zaman K: Oxidative stress as a mechanism of chronic cadmiuminduced hepatotoxicity and renal toxicity and protection by antioxidants. Toxicol Appl Pharmacol 1999;154:256-263.

50 Agarwal A, Balla J, Alam J, Croatt AJ, Nath KA: Induction of heme oxygenase in toxic renal injury - a protective role in cisplatin nephrotoxicity in the rat. Kidney Int 1995; 48:1298-1307.

51 Weinberg JM: The cell biology of ischemic renal injury. Kidney Int 1991;39:476-500. 